\title{
The usefulness of the totally intra-corporeal pringle maneuver with Penrose drain tube during laparoscopic left side liver resection
}

\author{
Young II Choi
}

\begin{abstract}
Department of Surgery, Kosin University Gospel Hospital, Kosin University College of Medicine, Busan, Korea
\end{abstract}
\begin{abstract}
Backgrounds/Aims: The Pringle maneuver is generally performed to reduce the amount of blood loss during hepatic resection. During laparoscopic liver resection, the Pringle maneuver can be used in several ways. We have developed a new Pringle maneuver (PM) with Penrose drain tube to sufficiently control blood loss during laparoscopic liver resection. This study was performed to determine the safety and outcome during laparoscopic left-sided hepatectomy performed using this new method. Methods: We describe the technique and results of the left-sided liver resection with totally intracorporeal PM with Penrose drain tube. We performed 37 laparoscopic left-sided hepatic resections with (PM group) or without the Penrose PM (No PM group). We retrospectively compared the short-term operative outcome between the No PM group $(n=12)$ and the PM group $(n=25)$ during laparoscopic left-sided liver resection. Results: Median PM duration was $34.3 \mathrm{~min}$. The median duration of the surgery using the totally intracorporeal PM with Penrose drain tube was $174 \mathrm{~min}$, while the surgical duration required for resection without the PM was 156 min. The median volume of operative blood loss was lower in the PM group than in the No PM group (No PM group (341 $\mathrm{ml})$ vs. PM group $(165 \mathrm{ml})$ ). There was no postoperative mortality and no open conversion. Conclusions: The totally intracorporeal PM with Penrose drain tube for laparoscopic hepatectomy is safe, reproducible, and can facilitate liver dissection during left-sided liver resection. (Ann Hepatobiliary Pancreat Surg 2020;24:252-258)
\end{abstract}

Key Words: Laparoscopy; Hepatectomy; Pringle maneuver

\section{INTRODUCTION}

Laparoscopic hepatectomy (LH) was introduced in 1996; since then, laparoscopic technology has evolved rapidly around the world. However, due to the associated technical complexity, the adoption of LH has been relatively slow, and many liver surgeons are still reluctant to perform it. Nevertheless, it has several advantages over open hepatectomy, which have led to an increase in the number of LHs performed worldwide. ${ }^{1-3}$

The previous studies shown that LH has significantly better results in terms of reduced blood loss, reduced postoperative hospitalization, improved cosmesis, better wound recovery, and early return to normal activity. ${ }^{4,5}$ These result were due to an improvement of surgical procedure and the development of device for LH. However, despite new tools developed for parenchymal transection, the main concern remains the bleeding control during hepatic resection. ${ }^{6-8}$ Furthermore, blood transfusion has been demonstrated to be a predictor of poor surgical results in liver resection with adverse effects on perioperative morbidity and mortality. ${ }^{9,10}$

Inflow blocking is one of the most effective methods to control bleeding during parenchymal transection. The Pringle maneuver (PM), first described in 1908 by Pringle, ${ }^{11}$ is a simple and easily reproducible method for inflow occlusion.

There are several reports on the use of the PM during LH. Here, we describe an easy, safe and unique way of performing totally intra-corporeal PM. More specifically, we describe a simple method for laparoscopic PM with Penrose drain tube which have been using at our center in most laparoscopic liver resections from 2018. This retrospective study was conducted to review our experience

Received: April 12, 2020; Revised: April 15, 2020; Accepted: April 17, 2020

Corresponding author: Young Il Choi

Department of Surgery, Kosin University College of Medicine, 262 Gamcheon-ro, Seo-gu, Busan 49267, Korea Tel: +82-51-990-6462, Fax: +82-51-246-6093, E-mail: tsojc@naver.com

Copyright (C) 2020 by The Korean Association of Hepato-Biliary-Pancreatic Surgery

This is an Open Access article distributed under the terms of the Creative Commons Attribution Non-Commercial License (http://creativecommons.org/ censes/by-nc/4.0) which permits unrestricted non-commercial use, distribution, and reproduction in any medium, provided the original work is properly cited. Annals of Hepato-Biliary-Pancreatic Surgery - pISSN: 2508-5778 - elSSN: 2508-5859 
with a easily obtainable penrose drain tube for laparoscopic PM and to investigate the safety of intermittent clamping during LH. We report the surgical results and improvements to the totally intra-corporeal PM using penrose drain tube. We also observed that the effective hepatic artery and portal blood flow blocking occurs while using this procedure.

\section{MATERIALS AND METHODS}

\section{Patient population and selection}

Between 2016 and 2019, we performed LH on 75 consecutive patients at Kosin University Gospel Hospital in Busan, Korea. Laparoscopic left-sided hepatectomy is the main criterion for this study (37 cases).

Our study population was divided into two groups: the No PM group with 12 patients and the PM with Penrose drain tube group with 25 patients.

\section{Surgical technique}

In this series, all patients underwent potentially curative hepatic resection with removal of gross pathologies with negative macroscopic margins. All procedures were performed by one experienced hepatobiliary surgeons throughout the study period.

The patients were placed in a supine and low lithotomy position, French position, with the surgeon standing between the patient's legs (Fig. 1). Five trocars were usually

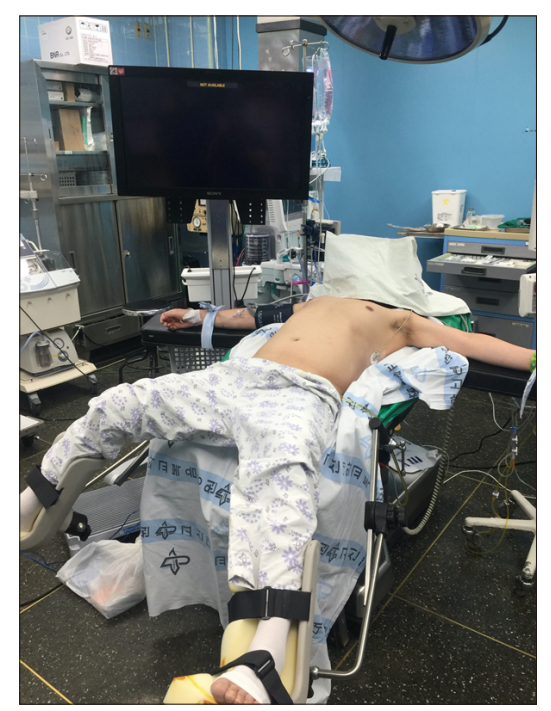

Fig. 1. Patient position, low lithotomy position, French position. used (Fig. 2). After the introduction of a 12-mm umbilical port using an open technique, continuous carbon dioxide pneumoperitoneum was induced at a pressure limit of 12 $\mathrm{mmHg}$ and flow of $6 \mathrm{~L} /$ minute to decrease the risk of gas embolism. The laparoscope was inserted through the supraumbilical $12 \mathrm{~mm}$ trocar site located 1 to $2 \mathrm{~cm}$ above the umbilicus. Five 5- to 12-mm trocars and a flexible, 3D laparoscope (ENDOEYE FLEX 3D, Olympus, Tokyo) were used. The liver was evaluated in all cases using intraoperative laparoscopic ultrasonography (Aloka Medical, Ltd.) to identify the hepatic veins and intrahepatic pathologies.

Subsequently, mobilization of the liver was initiated with the falciform ligament; the left lateral hepatic attachment and the triangular ligament were divided using an energy device (Harmonic scalpel HD 1000i, Ethicon EndoSurgery, Cincinnati, USA) after the round and falciform ligaments were dissected.

Next, the avascular lesser omentum was divided with electrocautery. From the port that was inserted at the upper mid line, Gold finger dissector (Blunt dissectors, Johnson \& Johnson, USA) with $15 \mathrm{~cm}$ sized Silicone Penrose drain tube (Sewoonmedical, Korea) was inserted from opened lesser omentum to pass behind the hepatoduodenal ligament (Fig. 3). The forcep was inserted until the Penrose drain tube reached the orifice that was in the Winslow's foramen (Fig. 4A). The edge of the drain tube was held with a forceps and was pulled out

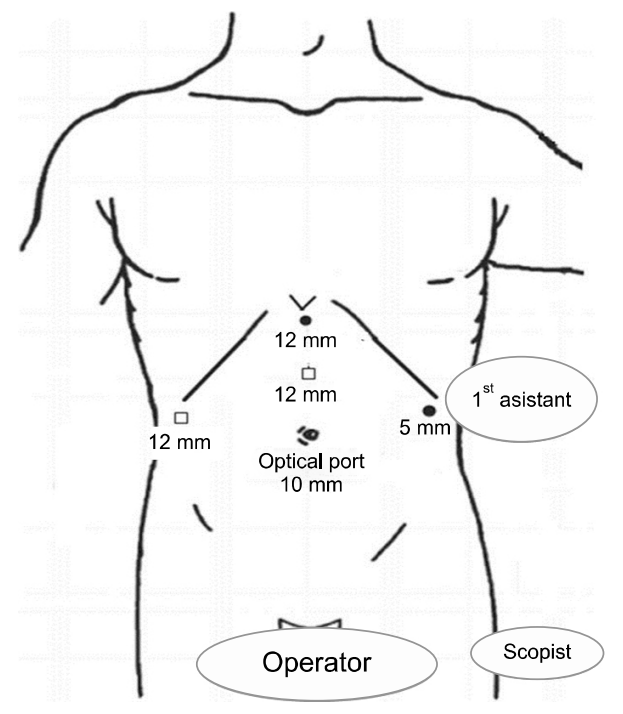

Fig. 2. The trocar location of laparoscopic hepatectomy. 
through the side hole of another edge of penrose drain tube for taping of the hepatoduodenal ligament; and finally, the penrose drain tube was pulled out of and lock with the Hemo-o-Lok clip (Weck Closure System,

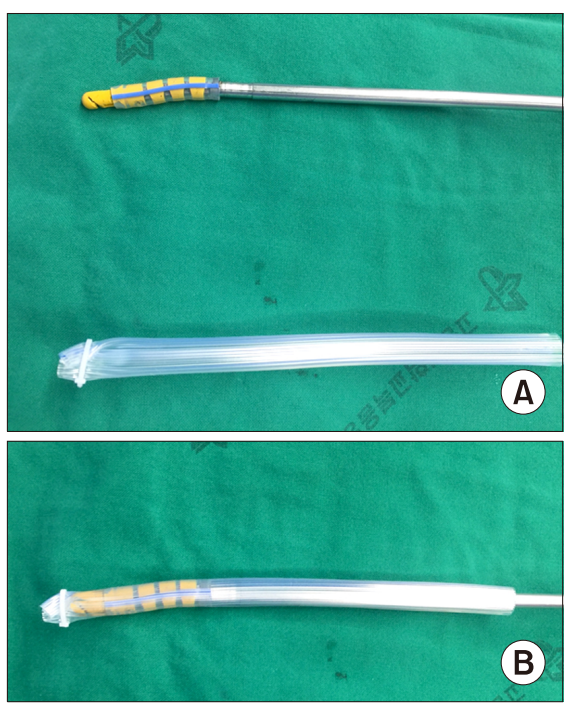

Fig. 3. (A) The length of Penrose drain tube is $15 \mathrm{~cm}$, Gold finger dissector. (B) Gold finger dissector in the Penrose drain tube.
Research Triangle Park, NC, USA) for use as a tourniquet to enable performance of a PM (Fig. 4B, C). Blood flow was occluded by clamping the Penrose drain tube with the Hemo-lock clip in order to perform a totally intracorporeal PM. Intermittent clamping was applied, with 15-minute clamping and 5-minute release periods.

Parenchymal transection was achieved using an energy device and Cavitron ultrasonic surgical aspirator (CUSA EXcel; Valleylab, Boulder, Colorado, USA) under totally intra-corporeal PM. Small vessels were ligated with clip or coagulated using an energy device. Intraparenchymal control of the major vessels was achieved with metal clips or Hemo-o-Lock clips, whereas major glissonean pedicle and major hepatic vein division was obtained with stapling devices. The resected specimen was placed in a plastic retrieval bag and removed through the slightly extended periumbilical incision.

\section{Statistical analysis}

Student's t-test, $\chi^{2}$ test, Fisher's exact probability test, and the Mann-Whitney $U$ test were used for analysis of parametric and non-parametric data, as appropriate. Statis-
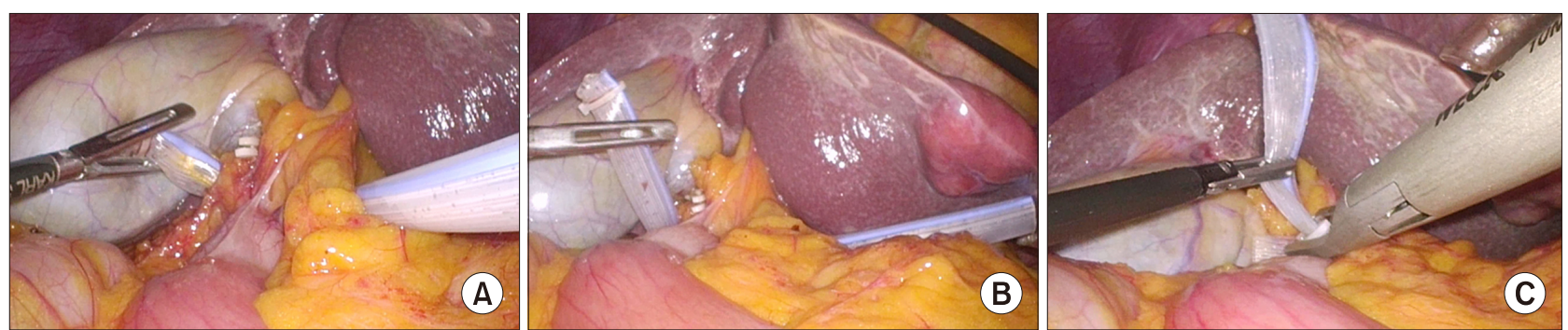

Fig. 4. Placement of totally intra-corporeal Pringle maneuver during laparoscopic hepatectomy. (A) The gold finger dissector with pnrose drain tube is inserted toward the direction of the lesser omentum. (B) The penrose drain is pulled out and passed behind the hepatoduodenal ligament. (C) The penrose drain tube is pulled out and lock with the Hemo-o-lok clip.

Table 1. Clinical characteristics of patients

\begin{tabular}{lccc}
\hline & No PM group $(\mathrm{n}=12)$ & PM group $(\mathrm{n}=25)$ & $p$ \\
\hline Male/female & $6 / 6$ & $14 / 11$ & 0.73 \\
Age (years) & $63(36-76)$ & $61(37-74)$ & 0.57 \\
BMI $\left(\mathrm{kg} / \mathrm{m}^{2}\right)$ & $22.4(18.7-27.5)$ & $3(12 \%)$ & 0.10 \\
ASA $\geq \mathrm{III}$ & $3(25 \%)$ & & \\
Liver disease & & 4 & \\
CRLM & 4 & 9 & \\
HCC & 4 & 2 & \\
IHCCC & 1 & 10 & \\
Others (bengin) & 3 & & \\
\hline
\end{tabular}

BMI, body mass index; ASA, American Society of Anesthesiology; CRLM, colorectal liver metastasis; HCC, hepatocellular carcinoma; IHCCC, intrahepatic cholangiocarcinoma 
Table 2. Operative characteristics and postoperative outcomes

\begin{tabular}{lccc}
\hline & No PM group $(\mathrm{n}=12)$ & PM group $(\mathrm{n}=25)$ & $p$ \\
\hline Type of hepatectomy & & & \\
$\quad$ Left hemihepatectomy & $7(58.3 \%)$ & $15(60.0 \%)$ & 0.69 \\
$\quad$ Left lateral sectionectomy & $5(41.7 \%)$ & $4(16 \%)$ & 0.41 \\
Colorectal combine resection & $3(25 \%)$ & $174(80-300)$ & 0.20 \\
Operative time (min) & $156(90-255)$ & $34.2(15-60)$ & 0.25 \\
Pringle time (min) & $341(20-1100)$ & $165(20-700)$ & \\
Estimated blood loss (ml) & 0 & $10.9(5-15)$ & \\
Intraoperative blood transfusion & $8.6(5-11)$ & 0 & \\
Hospital stay (days) & & 0 & \\
Postoperative morbidity & 1 & 0 & \\
Clavien-Dindo $\geq$ III & 0 & 0 & \\
Postoperative mortality & & & \\
Open conversion &
\end{tabular}

${ }^{\mathrm{a}}$ Exclude colorectal combine resection

tical analyses were performed using SPSS ver. 25.0 (IBM, New York, NY, USA). Differences of $p<0.05$ were considered significant.

\section{RESULTS}

From 2016 to 2019, a total of 75 patients underwent laparoscopic liver resection. Among these 75 patients, laparoscopic left-sided hepatectomy was performed in 37 $(49.3 \%)$ patients. There were 12 cases in the no PM group and 25 cases in the PM group. During the study period, there was no morbidity associated with placement of the penrose drain tube and no operative mortality.

The clinical characteristics of patients are shown in Table 1. The median patient age was 63 years (range 36-76), and $20(54.0 \%)$ patients were men. Patients demographics including sex, age, body mass index (BMI), and American Society for Anesthesiologists (ASA) physical status were similar between two groups.

Table 2 summarize the perioperative outcomes between no PM group and PM group. In the no PM group, 12 patients underwent laparoscopic left side liver resection, including 6 left hemihepatectomy, 5 left lateral sectionectomy. In the PM group, 25 patients underwent laparoscopic left side liver resection, including 15 left hemihepatectomy, 10 left lateral sectionectomy. The mean operative time was $156 \mathrm{~min}$ in no PM group and $174 \mathrm{~min}$ in PM group. The estimated blood loss was higher in no PM group (341 (20-1100) ml vs. 165 (20-700) $\mathrm{ml}, p=$ 0.20 ), but there were not significantly different between

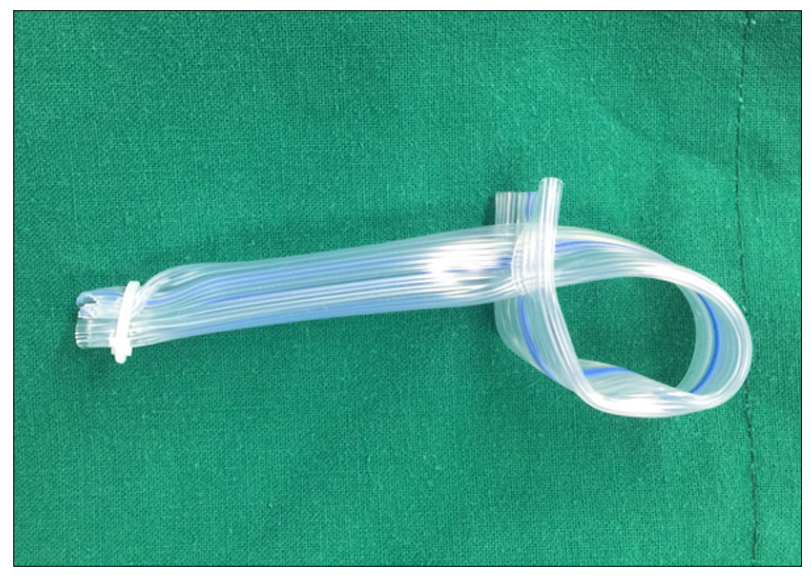

Fig. 5. The edge of Penrose drain tube was held with a forceps and was pulled out through the side hole of another edge of Penrose drain tube for taping of the hepatoduodenal ligament.

groups. There was no blood transfusion during surgery in both group. The mean hospital stay was 8.6 day in no PM group and 10.9 day in PM group. There was one procedure that require surgical exploration after surgery in no PM group, the case was for anastomotic leakage from colorectal anastomosis site. There was no open conversion and no mortality in both group.

\section{DISCUSSION}

LH is a safe and effective treatment that is associated with improved short-term outcomes compared to the open technique. ${ }^{12-15}$ Additionally, LH is comparable to open hepatectomy regarding long-term oncological benefits. ${ }^{15,16}$ 
Therefore, progression has been made from simple hepatectomy to major hepatectomy and even laparoscopic donor hepatectomy.

Nevertheless, control of bleeding remains the major concern during parenchymal transection. PM is a traditional and the most commonly used method to decrease blood loss during open liver resection its effectiveness and simplicity. In laparoscopic surgery, various methods for applying PM have been published. ${ }^{7,11,17-20}$

Some authors have introduced their extra-corporeal PM technique during LH and Robotic hepatectomy. They suggested that compared with the intra-corporeal PM technique, the extra-corporeal PM technique confers the following advantages: it combines all the well-known advantages of the technique used in open operations (ie it is safe, quickly usable in case of bleeding, and achieves complete inflow occlusion). This seems advantageous in that the absence of tactile feedback can lead to serious vascular structural damage during inflow occlusion due to the clamp force applied to the pedicle. However, due to the additional port for PM, it is likely to interfere with the docking and undocking of the robot arm during robot liver surgery, and it is also interfere with the instrumental forcep movement during LH. We assume the advantages and drawbacks of each technique are experience-based and not evidence-based. One of the potential drawbacks of the use of an additional trocar for extra-corporeal PM (compared with intra-corporeal PM) is the risk of gas leakage from the abdominal cavity. ${ }^{21-23}$

Totally intra-corporeal PM technique also has several methods. ${ }^{24,25}$ This report demonstrates an easy way to perform a new totally intra-corporeal PM during LH using simple and readily available materials without tourniquet and additional port. Our approach to hepatectomy includes low central venous pressure (CVP) anesthesia and liberal use of PM when bleeding, even minor, occurs during transection. For this reason, before we begin hepatic parenchymal transection, penrose drain tube is passed routinely around the hepatoduodenal ligament, except when taping is impossible due to adhesion. The pedicle clamping is used on demand according to surgeon's preference and local conditions. This technique is easy and dose not require any special instruments. Our policy of intermittent 15 min clamping, 5 min unclamping is based on practical experience. We have found that the association of PM with low CVP and pneumoperitoneum pressure achieves a bloodless field in most instances.

Our technique has several advantages. It is simple, reliable and reproducible. Due to the fact that it is fully intracorporeal, our technique does not require a working port or placement of an additional port. Its intra-corporeal position is flexible and therefore it does not interfere with the surgeons' view and actions. Clamping and unclamping can be performed in few seconds, including in emergency situations. It is cost-effective since it does not require any expensive components. Komeda et al. ${ }^{26}$ suggested Endo intestinal clips for intra-corporeal PM during LH. The benefits of using an Endo intestinal clip are that additional port for PM dose not need to be occupied, and gripping force can be maintained at a steady level. However, with this method, complete occlusion of the inflow through thick and fatty hepatoduodenal ligament is difficult to achieve. A further advantage of our method is the of achieving complete occlusion, by completely surrounding the hepatoduodenal ligament, especially when the porta hepatis is thick or fatty (Fig. 5).

A potential limitation of our technique is that there should be no adhesions to allow the Penrose drain tube to pass through the Winslow foramen between the hepatoduodenal ligament and the inferior vena cava. Howev-
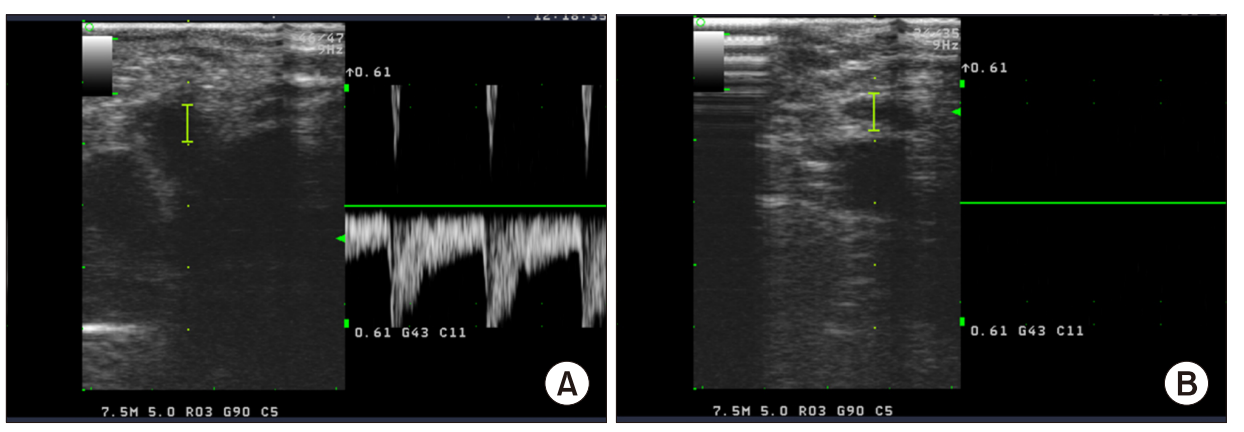

Fig. 6. (A) Doppler ultrasonography. Hepatic artery flow before occlusion with Penrose drain tube clamp. (B) Doppler ultrasonography. Disappeared hepatic artery flow after occlusion with Penrose drain tube clamp. 
er, such adhesions are rare in the absence of previous surgery, but not uncommon in repeat hepatectomy or upper abdominal surgery, such as right colectomy or even a simple cholecystectomy.

Our study is limited by the small number of patients. Additionally, no objective measurements were performed to assess the degree of clamping by intraoperative Doppler ultrasonography or pressure measurements of the Penrose drain. Although not applicable to all patients during $\mathrm{LH}$, the same PM was applied during open and laparoscopic liver surgery. Although it is difficult to measure the exact quantitative pressure of our PM, Doppler ultrasonography allowed us to observe that the blood flow to the liver disappeared completely after our PM (Fig. 6). It seems to have a study on the minimum compression pressure to laparoscopic PM tool for achieving complete flow blocking effect.

In coclusion, the totally intracorporeal PM with Penrose drain tube may be effective in reducing intraoperative blood loss during laparoscopic left-sided hepatectomy, although the difference is not statistically significant. Moreover, our method is easy, reproducible, effective, safe, can be implemented quickly, and allows intermittent clamping during LH. The current experience-based analysis remains to be confirmed with prospective randomized studies.

\section{CONFLICT OF INTEREST}

The authors have no conflict of interest.

\section{ORCID}

Young Il Choi: https://orcid.org/0000-0002-9630-6287

\section{AUTHOR CONTRIBUTIONS}

Conceptualization: YIC. Data curation: YIC. Formal analysis: YIC. Funding acquisition: YIC. Methodology: YIC. Project administration: YIC. Visualization: YIC. Writing - original draft: YIC. Writing - review \& editing: YIC.

\section{REFERENCES}

1. Azagra JS, Goergen M, Gilbart E, Jacobs D. Laparoscopic ana- tomical (hepatic) left lateral segmentectomy-technical aspects. Surg Endosc 1996;10:758-761.

2. Buell JF, Thomas MJ, Doty TC, Gersin KS, Merchen TD, Gupta $\mathrm{M}$, et al. An initial experience and evolution of laparoscopic hepatic resectional surgery. Surgery 2004;136:804-811.

3. Chen J, Li H, Liu F, Li B, Wei Y. Surgical outcomes of laparoscopic versus open liver resection for hepatocellular carcinoma for various resection extent. Medicine (Baltimore) 2017;96:e6460.

4. Inoue Y, Hayashi M, Tanaka R, Komeda K, Hirokawa F, Uchiyama K. Short-term results of laparoscopic versus open liver resection for liver metastasis from colorectal cancer: a comparative study. Am Surg 2013;79:495-501.

5. Ciria R, Ocaña S, Gomez-Luque I, Cipriani F, Halls M, Fretland $\AA \mathrm{A}$, et al. A systematic review and meta-analysis comparing the short- and long-term outcomes for laparoscopic and open liver resections for liver metastases from colorectal cancer. Surg Endosc 2020;34:349-360.

6. Corvera CU, Dada SA, Kirkland JG, Garrett RD, Way LW, Stewart L. Bipolar pulse coagulation for resection of the cirrhotic liver. J Surg Res 2006;136:182-186.

7. Aldrighetti L, Pulitanò C, Arru M, Catena M, Finazzi R, Ferla G. "Technological" approach versus clamp crushing technique for hepatic parenchymal transection: a comparative study. J Gastrointest Surg 2006;10:974-979.

8. Chang YC, Nagasue N, Chen CS, Lin XZ. Simplified hepatic resections with the use of a Chang's needle. Ann Surg 2006; 243:169-172.

9. Schiergens TS, Rentsch M, Kasparek MS, Frenes K, Jauch KW, Thasler WE. Impact of perioperative allogeneic red blood cell transfusion on recurrence and overall survival after resection of colorectal liver metastases. Dis Colon Rectum 2015;58:74-82.

10. Bennett S, Baker LK, Martel G, Shorr R, Pawlik TM, Tinmouth A, et al. The impact of perioperative red blood cell transfusions in patients undergoing liver resection: a systematic review. HPB (Oxford) 2017;19:321-330.

11. Pringle JH. V. Notes on the arrest of hepatic hemorrhage due to trauma. Ann Surg 1908;48:541-549.

12. Belli G, Fantini C, D'Agostino A, Cioffi L, Langella S, Russolillo $\mathrm{N}$, et al. Laparoscopic versus open liver resection for hepatocellular carcinoma in patients with histologically proven cirrhosis: short- and middle-term results. Surg Endosc 2007;21: 2004-2011.

13. Yoon YI, Kim KH, Kang SH, Kim WJ, Shin MH, Lee SK, et al. Pure laparoscopic versus open right hepatectomy for hepatocellular carcinoma in patients with cirrhosis: a propensity score matched analysis. Ann Surg 2017;265:856-863.

14. Jiang S, Wang Z, Ou M, Pang Q, Fan D, Cui P. Laparoscopic versus open hepatectomy in short- and long-term outcomes of the hepatocellular carcinoma patients with cirrhosis: a systematic review and meta-analysis. J Laparoendosc Adv Surg Tech A 2019;29:643-654.

15. Sotiropoulos GC, Prodromidou A, Kostakis ID, Machairas N. Meta-analysis of laparoscopic vs open liver resection for hepatocellular carcinoma. Updates Surg 2017;69:291-311.

16. Kim KH, Kang SH, Jung DH, Yoon YI, Kim WJ, Shin MH, et al. Initial outcomes of pure laparoscopic living donor right hepatectomy in an experienced adult living donor liver transplant center. Transplantation 2017;101:1106-1110.

17. Huntington JT, Royall NA, Schmidt CR. Minimizing blood loss during hepatectomy: a literature review. J Surg Oncol 2014;109: $81-88$.

18. Otsuka Y, Kaneko H, Cleary SP, Buell JF, Cai X, Wakabayashi G. What is the best technique in parenchymal transection in laparoscopic liver resection? Comprehensive review for the clinical 
question on the 2nd International Consensus Conference on Laparoscopic Liver Resection. J Hepatobiliary Pancreat Sci 2015;22:363-370.

19. Otsubo T, Takasaki K, Yamamoto M, Katsuragawa H, Katagiri $\mathrm{S}$, Yoshitoshi K, et al. Bleeding during hepatectomy can be reduced by clamping the inferior vena cava below the liver. Surgery 2004;135:67-73.

20. Piardi T, Lhuaire M, Memeo R, Pessaux P, Kianmanesh R, Sommacale D. Laparoscopic Pringle maneuver: how we do it? Hepatobiliary Surg Nutr 2016;5:345-349.

21. Dua MM, Worhunsky DJ, Hwa K, Poultsides GA, Norton JA, Visser BC. Extracorporeal Pringle for laparoscopic liver resection. Surg Endosc 2015;29:1348-1355.

22. Lim C, Osseis M, Lahat E, Azoulay D, Salloum C. Extracorporeal Pringle maneuver during laparoscopic and robotic hepatectomy: detailed technique and first comparison with intra- corporeal maneuver. J Am Coll Surg 2018;226:e19-e25.

23. Mizuguchi T, Kawamoto M, Nakamura Y, Meguro M, Hui TT, Hirata K. New technique of extracorporeal hepatic inflow control for pure laparoscopic liver resection. Surg Laparosc Endosc Percutan Tech 2015;25:e16-e20.

24. Laurenzi A, Cherqui D, Figueroa R, Adam R, Vibert E, Sa Cunha A. Totally intra-corporeal Pringle maneuver during laparoscopic liver resection. HPB (Oxford) 2018;20:128-131.

25. Huang JW, Su WL, Wang SN. Alternative laparoscopic intracorporeal Pringle maneuver by Huang's loop. World J Surg 2018;42:3312-3315.

26. Komeda K, Hayashi M, Inoue Y, Shimizu T, Asakuma M, Hirokawa $\mathrm{F}$, et al. Clinical usefulness of endo intestinal clips during Pringle's maneuver in laparoscopic liver resection: a technical report. Surg Laparosc Endosc Percutan Tech 2013;23: e103-e105. 